\title{
A Nature survey of the neurosciences
}

In one sense, the neurosciences are at the cutting edge of the new biology. Informed by new understanding and empowered with a staggering array of new techniques, they are engaged with one of the most teasing - and oldest - questions - "How does the physical representation of the mind, which is called the brain, carry out its functions?'”. But in another sense, the neurosciences include some of the most tedious of investigations. The continual improvement of technique, staggering though it may be, is as often a source of frustration and disappointment as of discovery. This year's new way with neurones could be next year's discard.

Single experiments, such as investigations of the behavioural consequences of supposedly neurone-specific drugs or the consequences for neuronal development of particular forms of sensory deprivation, may take weeks or months. Often, the behaviour of neurones in, say, the human central nervous system must be inferred from that of model systems. (There are subliminal as well as formal inhibitions of the use of human brains.) In general, the neurosciences are in the business of making bricks without straw ${ }^{I}$.

A further difficulty is the huge gap between the microscopic and the macroscopic. In an organ such as the liver (admittedly at one extreme), the biochemistry of a single cell is a good approximation to that of the whole. In the nervous system, however, cells with outwardly indistinguishable properties must in reality have different functions. The neurosciences are thus a challenge for the reductionists: can it be respectable to account for the properties of an integrated nervous system by giving a catalogue of neurones with different properties? The outward similarities of cells from nervous tissue have prompted an often exciting hunt for differences between them; the differentiation of a supposedly common cell type in such subtly different ways is, for the time being, neglected.

So why do the neurosciences remain buoyant? Part of the explanation is that the frustrations of laboratory work and of concept formation are more than outweighed by the underlying challenge. How, after all, does the brain work? Nobody knows, but many believe, as their predecessors have always thought, that they are within an ace of knowing.
What follows is an account, necessarily subjective, of where the neurosciences stand in the opinion of outside observers. It has been compiled partly by the reading of the journals Nature not dominant but not negligible among them) but mostly by conversation.

The objective is not so much to startle neurobiologists themselves as to interest those who work in other fields in what their fellowscientists are up to. For one of the abiding problems of the neurosciences is that they are poorly understood.

The techniques usually demand exceptional skill. The jargon is more than usually impenetrable because it has grown up by accretion from several disciplines - and with plenty of etymological licence: thus while a chemical that offsets the effects of some drug may properly be called its antagonist, why should another chemical with similar effects be called an agonist when no such word appears in English dictionaries (and the original Greek has a different meaning)? Then, like the spectroscopists of the early 1920s, the neurobiologists often give the impression of grubbing for data while lacking the leisure to consider the grand questions - what is it all for? and so on. The prizes will go, no doubt, to those who ask these questions.

Indeed, when asked what their long-term objectives are, most people say that philosophical speculations are the prerogative of retired persons, many of whom have been known to write books on the subject in their old age. Could it be that the neurosciences as they are would profit from a greater reflectiveness among their practitioners? Is it possible that in this field, as in high-energy physics a few years ago, something that might be called truth is obscured by too much data? Or does that opportunity still lie some way ahead?

Nobody can tell. Since the beginning, say in Galvani's time, the most serious impediment to the neurosciences has been the difficulty of forming concepts. The recent influx of people from computer science - some of whom like to say they work in artificial intelligence - has helped a little, but not decisively. Maybe the outstanding problem of the neurosciences is to specify the problem to be solved. 